\title{
La clínica de higiene mental infantil en Brasil. La psicología como recurso
}

\author{
The Child Mental Hygiene Clinic in BRAZIL. Psychology as a Resource
}

Recibido: diciembre 13 de 2013 | Revisado: febrero 2 de 2014 | Aceptado: julio 17 de 2014

\author{
Roselania Francisconi Borges* \\ MARIA LUCIA BOARINI \\ Universidade Estadual de Maringá, Paraná, Brasil
}

doi: 10.11144/Javeriana.upsy13-5.lchm

Para citar este artículo: Borges, R. F., \& Boarini, M. L. (2014). La clínica de higiene mental infantil en Brasil. La psicología como recurso. Universitas Psychologica, 13(5), 1697-1708. http://dx.doi. org/10.11144/Javeriana.upsy13-5.lchm

E-mail: roselaniafborges@uol.com.br

\section{RES UMEN}

Este estudio tuvo el objetivo de demostrar que los integrantes de la Liga Brasileña de Higiene Mental -en su mayoría médicos, educadores y juristas- contribuyeron a la inserción de los saberes psicológicos en Brasil, a principios del siglo XX. Teniendo como perspectiva teórica el análisis histórico y como fuente primaria los Arquivos Brasileiros de Higiene Mental, en su periódico oficial, se realizó una investigación bibliográfica para demostrar que este grupo encontró en los instrumentos de la psicología, entre ellos, las pruebas psicológicas, una forma de explicar las diferencias individuales. Los presupuestos de la psicología fueron tomados como base científica para tornar la higiene mental un medio de saneamiento psíquico de la población. $\mathrm{Al}$ crear la Clínica de Euphrenia, destinada a realizar la psicohigiene del niño, abrieron campos para inserción de la psicología en la educación y en la salud, con la profilaxis mental de la infancia como objetivo.

Palabras clave

higiene mental; higienismo; historia de la psicología en Brasil

\section{A B S T R A C T}

The objective of this study was to demonstrate that members of the Brazilian Mental Hygiene League - most of whom were physicians, educators and jurists - contributed to introduce knowledge in psychology to Brazil during the early $20^{\text {th }}$ century. A bibliographical research study was carried out using historical analysis as theoretical perspective and featuring the Brazilian Mental Hygiene Archives - official publication of the League - as primary source. The aim was to demonstrate that the group found a way to explain individual differences from the instruments of psychology, including psychological tests. The assumptions of psychology were taken as scientific support to transform mental hygiene into a vehicle for mental healing of the population. By opening the Euphrenic Clinic to conduct psycho-hygiene of children, they created openings to insert psychology into the fields of education and health, with the goal of performing mental prophylaxis the population, particularly the children.

Keywords

mental hygiene; hygienism; history of psychology in Brazil 


\section{Introducción}

En este inicio de siglo XXI, en Brasil, podemos identificar la implementación de diversos servicios de salud mental, dirigidos a los cuidados de la infancia y la adolescencia. Tales servicios tienen soporte legal en el Decreto № 336 (2002) del Ministerio de la Salud que dispone las reglas de funcionamiento del Centro de Atención Psicosocial Infantojuvenil (CAPSi) como un "servicio ambulatorio de atención diaria destinado a niños y adolescentes con trastornos mentales". Para atender a esta población, el psicólogo está entre los profesionales indicados para conformar el equipo técnico necesario para ofertar una atención especializada y calificada.

A primera vista, tanto la creación de este servicio como la participación del psicólogo en el equipo técnico puede tomarse como algo del momento presente, constituyéndose una novedad que, por la óptica de la inserción en el mercado de trabajo, abre un nuevo campo de actuación para el psicólogo.

De hecho, oficialmente la implantación de esta modalidad de servicio de atención a la infancia abrió nuevas posibilidades de trabajo para el psicólogo en cuanto profesional y para la psicología como profesión reconocida en Brasil hace algunas décadas, más precisamente en 1962 por la Ley 4.119 (1962). En términos cuantitativos, según datos del Ministerio de la Salud, hasta 2011 habían sido implementados 149 Centros de Atención Psicosocial Infantojuvenil en Brasil (2012).

No obstante, la actuación del psicólogo, como práctica o herramienta de trabajo dirigida a las cuestiones de la infancia, puede ubicarse en períodos anteriores, y en el inicio del siglo XX, en Brasil, en las actividades de estudios, investigaciones e intervenciones en la sociedad realizadas por los integrantes de la Liga Brasileña de Higiene Mental (LBHM). En este caso, al recuperar los trabajos desarrollados por los integrantes de la LBHM podemos identificar acciones coincidentes con la instauración de la psicología científica en Brasil.

Si tomamos como parámetro el origen de las ideas psicológicas en Brasil, Massimi (2001) señala la emergencia de conceptos acerca de los fenómenos psicológicos formulados en el ámbito de la literatura espiritual y de la producción filosófica de la Compañía de Jesús, a lo largo de los siglos XVI y XVII. Sin embargo, tales estudios poseían una dimensión filosófica que escapaban al carácter científico que fue configurándose, poco a poco, en el inicio del siglo XX. Este carácter se fue produciendo con la contribución de los estudios e investigaciones que tenían la higiene mental y la eugenesia, como nuevas tecnologías formuladas y transmitidas por el movimiento higienista.

Así, en las primeras décadas del siglo XX, los higienistas se valieron de la ciencia psicológica como soporte teórico-práctico para sus acciones e intervenciones en la sociedad, al mismo tiempo ejercieron gran influencia en la psicología científica en Brasil. Esto es lo que afirma Massimi (1990, p. 64 , resaltado en el texto) al enfatizar la existencia de "figuras relevantes de profesionales e investigadores en el campo médico que dedican su actividad a estudios psicológicos relacionados a la neurología, a la psiquiatría, a la higiene mental y a la criminología y psiquiatría forense, teniendo el objetivo de contribuir para la creación de una 'ciencia del hombre' como un todo".

También es importante constatar que con el pasar del tiempo se fortaleció la creencia en la vertiente de la eugenesia como solución para las enfermedades sociales, pero, de la misma forma, fue motivo de divergencia de opiniones entre aquellos que condenaban las medidas de selección hereditaria como supuesta garantía de un futuro exento de enfermedades transmitidas por la herencia, como indican los clásicos estudios de Costa (1989) y Reis (2003).

Como tal producción es muy vasta y abarcadora, por cuestiones metodológicas y, dentro de los límites de este estudio, abordaremos los estudios e investigaciones dirigidos solo a la temática de la infancia. Sobre ello, inumerables ejemplos pueden confirmar que este grupo de intelectuales tenía, hace por lo menos un siglo, entre sus muchas preocupaciones, las cuestiones del niño, considerado portador de trastornos mentales, degenerado, incorregible, delincuente o criminal, conforme Mendonça (2006), Zaniani y Boarini (2011), Feitosa (2012), entre otros. 
En un estudio que data de 1925, el Dr. Waclaw Radecki (1887-1953)1 ${ }^{1}$, director del Laboratorio de Psicología en Colônia dos Alienados en el barrio de Engenho de Dentro, abogando sobre la necesidad de promover la higiene mental del niño "basada sobre las leyes de la psicología", da orientaciones sobre las peculiaridades del período de la infancia, enfatizando que "en el período infantil su evolución difiere, cualitativamente de la psicología del hombre adulto" y que:

(...) el niño no es un hombre adulto en miniatura, pero representa período cualitativamente diferente, donde las leyes fundamentales de la psicología general, no perdiendo su valor, se refieren, no obstante, a otras funciones que no las que se encuentran en el hombre adulto. (Radecki, 1925, p. 12, subrayado nuestro)

En otro estudio de 1930, el presidente de la LBHM, Dr. Ernani Lopes, discute la cuestión de los considerados menores incorregibles -que desde el punto de vista intelectual eran denominados retrasados, deficientes intelectuales o débiles mentales, y del punto de vista moral denominados menores antisociales-que para él, eran de hecho, "los verdaderos, los legítimos menores incorregibles" (Lopes, 1930a, p. 242).

Otra preocupación expresada por el mismo autor se refería a las psicosis infantiles en la perspectiva de que la "medicina mental para las primeras edades" podría auxiliar la detección de trastornos que se manifestarían solamente en la fase adulta. Defendiendo tal cuestión afirma,

1 Nacido en Polonia, emigró al Brasil y se estableció en Paraná, en 1923. Se trasladó a Rio de Janeiro para trabajar con Gustavo Riedel, en la fundación de un Laboratorio de Psicología que fue instalado en 1924 en la Colonia de Psicópatas, en Engenho de Dentro. Este laboratorio pasó a funcionar bajo su dirección. En 1932, debido al tamaño que había alcanzado, el Laboratorio fue transformado en Instituto de Psicología por el Decreto Ley № 21.173 del Estado de Rio de Janeiro. En 1933, se trasladó a Montevideo, Uruguay, donde vivió hasta su muerte en 1953. En este período fundó el Centro de Estudios Psicológicos de Buenos Aires, en Argentina, y el Centro de Estudios Psicológicos de Montevideo, habiendo recibido la misión del gobierno uruguayo de introducir en aquel país las bases de la enseñanza de psicología con el modelo que ya había en Europa. Para obtener más datos sobre la trayectoria de Waclaw Radecki en América Latina, se puede onsultar a Centofanti (1982).
En la era clínica contemporánea, caracterizada sobre todo por la preocupación del diagnóstico precoz, ya no satisface a los especialistas el mero estudio de las psicosis de la infancia. Se comienza en fin a comprender el alto interés de sorprender, ya en la infancia, ciertas psicosis del adulto, rastreables con gran antelación por sus leves manifestaciones prodrómicas o incipientes. (Lopes, 1930a, p. 246, subrayado del autor seguido de subrayado nuestro)

Con tales ejemplos no estamos aquí haciendo una argumentación o defensa de una "degeneración" de la infancia, que viene de larga data, sino buscando apuntar que en Brasil, hace por lo menos un siglo, la preocupación por la infancia "normal" y "anormal" era una de las inquietudes de un grupo de profesionales, denominados por ellos mismos de higienistas, que emprendían esfuerzos para implantar los ideales de la higiene mental en suelo brasileño.

En este caso, nuestro objetivo es demostrar que este grupo de intelectuales -en su mayoría formada por médicos, escritores, juristas y educadores- con sus estudios sobre diversas temáticas respecto de la vida en sociedad (inmigración, toxicomanía, trabajo, enfermedad mental, delincuencia, alcoholismo, suicidio, educación sexual, pruebas psicológicas, entre otros), a partir de diversos segmentos de la sociedad (niños, mujeres, indígenas, soldados, enfermos mentales, alcohólicos, entre otros) contribuye grandemente a demarcar el principio de la historia de la psicología científica en Brasil, dando el color y el tono de las ideas psicológicas transmitidas y producidas en aquel momento histórico. Sin embargo, esta historia es, en general, ignorada en estudios sobre la atención a la infancia, pero ampliamente contemplada en los Arquivos Brasileiros de Higiene Mental. Debido a ello, en nuestro entender, es necesario recuperar esta historia.

Tenemos por evidencia histórica, a partir de los trabajos de la LBHM, transmitida principalmente por medio de su periódico oficial Arquivos Brasileiros de Higiene Mental (ABHM) -editado de 1925 a 1947- que sus integrantes contribuyeron a la inserción de la psicología como ciencia en suelo brasileño, antes de su institucionalización. Muchos 
de estos integrantes actualmente son reconocidos como precursores de la psicología en Brasil, entre ellos, Manoel José do Bomfim (1868-1932), Artur Moncorvo Filho (1871-1944), Plínio Olinto (18861956), Júlio Pires Porto-Carrero (1887-1937), Antonio Carlos Pacheco y Silva (1898-?), Arthur Ramos (1903-1949). Es importante señalar que los estudios sobre el psicoanálisis también primaban entre los intereses y las producciones de los higienistas. Sobre esta temática, los médicos Arthur Ramos y Júlio Pires Porto-Carrero, entre otros, pueden ser considerados importantes pioneros (Mendonça, 2006).

Estos y otras decenas de personalidades de la historia de la psicología en Brasil son homenajeados en el Dicionário biográfico da psicologia no Brasil, editado por el Consejo Federal de Psicología en 2001. Esta obra, producida por el Grupo Interinstitucional de Investigación en Historia de la Psicología, tuvo el objetivo de "registrar la contribución de profesionales, pensadores e investigadores a la construcción del área de la Psicología en Brasil, en el período anterior a la creación de los cursos universitarios y a la institucionalización de la profesión de psicólogo" (Campos, 2001, p. 5). Por sus contribuciones en diversas áreas del conocimiento, en general, son reconocidos en varias publicaciones como pioneros de la educación, medicina y psicología como ciencia aplicada; sin embargo, son poco citados como integrantes del movimiento higienistas y miembros de la LBHM.

\section{El pensamiento higienista y la psicología}

La propuesta de rescatar el "encuentro" entre el pensamiento higienista y la psicología puede traer algunas dificultades de orden interpretativo, las cuales demandan algunas explicaciones. Con esto, estamos queriendo afirmar que al destacar ideas producidas en determinado tiempo histórico tenemos que, necesariamente, atenernos al contexto en el cual fueron producidas. En este caso, destacamos la indispensable "utilización de una lente que sea capaz de verlos en su tempo histórico" bajo pena de que no superemos "aquellas explicaciones que nos paralizan, a la medida que naturalizan la vida humana y las relaciones sociales" (Boarini, 2012, p. 22 , subrayado de la autora).
En esta perspectiva, es importante destacar que la preocupación de estos "hombres de ciencia” (Caldas, 1929) no surgió por casualidad y sin propósito definido. Igualmente, se configuró, en el inicio del siglo XX, en el auge de la República, con el cambio del modelo de producción y las posibilidades de industrialización, en tanto necesidad de invertir en las nuevas generaciones, teniendo la familia y la escuela como algunos de sus principales enfoques de intervención, bajo la perspectiva de constituir personas vigorosas y saludables con el fin de convertir al Brasil en una gran nación. En este sentido, realizar el propósito de promover la profilaxis mental de la población brasileña se tornó una incumbencia cabal para los integrantes de la LBHM, en un país que tenía el progreso como su principal meta para crecer y proyectarse en el escenario sociopolítico y económico mundial. Por lo tanto, la higiene mental de la población fue uno de los caminos elegidos.

Buscando alcanzar este ideal, los higienistas tuvieron en las ideas psicológicas producidas en la época un campo fértil para ampliar sus estudios psicológicos sobre la infancia, tanto en el sentido de corroborar el pensamiento vigente o también para superar el conocimiento producido hasta entonces, contribuyendo a la producción de nuevas concepciones sobre esta temática.

Las palabras del Dr. J. P. Fontenelle, docente de la asignatura de Hygiene de la Escuela Normal del Distrito Federal, puede ilustrar esta afirmación:

La base científica de la higiene mental es la psicología, de la misma forma que la psicología es el fundamento de la salud física, conviene siempre recordar la interdependencia de las dos esferas de actividad y las reacciones de una sobre la otra. La psicología nos muestra cómo el sistema nervioso responde a los estímulos externos y permite el perfecto ajuste del organismo a las condiciones del medio, por intermedio de impulsos que llevan a la acción conveniente, sea refleja o inconsciente, sea voluntaria o elegida. (1925, p. 2, subrayado nuestro)

En esta perspectiva, los problemas relativos al proceso salud y enfermedad mental eran tomados 
como fenómenos naturales concernientes al organismo y a las experiencias sociales que, cuando no eran desarrollados de forma espontánea y saludable, deberían ser corregidos, pues "la mentalidad desarrollada correctamente, $\mathrm{y}$ actuando por medio de reacciones convenientes, se traduce en el comportamiento más adecuado al ajuste del individuo al medio físico y social, exhibiendo lo que se puede llamar la salud mental" (Fontenelle, 1925, p. 3, subrayado del autor).

En función de esto, la importancia atribuida a la higiene mental del niño por la vía de la psicología, se destacaba en los diversos escritos de los higienistas brasileños. También se establecía el intercambio intelectual entre los países, con lo cual se ampliaba la posibilidad de ofrecer y recibir conocimientos. En un evento realizado en Montevideo el 6 de noviembre de 1931, por indicación del Dr. Belisário Penna, Ministro de la Educación y Salud Pública, el Dr. Ernani Lopes dictó una conferencia intitulada "La higiene mental y la psicología en Brasil", en la cual señaló que los higienistas brasileños se interesaban por los estudios de los uruguayos sobre psicología y que la biblioteca de la LBHM poseía "libros y revistas uruguayas de psiquiatría, antialcoholismo y psicopedagogía desde su fundación, hace como cuatro años. Por esto digo que los neurohigienistas ya hacemos el intercambio intelectual en nuestra especialidad" (Lopes, 1932, p. 138).

En esta palestra, Lopes inclusive destaca las actividades del Laboratorio de Psicología Aplicada de la LBHM, adjunto a la Clínica Psiquiátrica de la Facultad de Medicina de Rio de Janeiro, y explica que además "en ciertas provincias de Brasil se observa hoy un desarrollo de la especialidad psicológica sin duda de los más promisores. Son ellas sobre todo: San Paulo, Bahia, Pernambuco, Minas Gerais y Rio Grande do Sul". En seguida, describe algunas de las peculiaridades de cada localidad en relación con los trabajos de psicología, como siendo "investigaciones de alto mérito" que, en líneas generales, consistía en la evaluación por medio de la utilización de pruebas psicométricas a partir de escalas elaboradas en otros países y de la adaptación de estas escalas a la realidad brasileña. En el caso de Pernambuco, Ernani Lopes destacaba que una actividad digna anotar era:
(...) la excelente adaptación brasileña del test de las 100 cuestiones de Ballard por Ulysses Pernambucano y Anita Paes Barreto, trabajo editado el año pasado por nuestra Liga y del cual presento aquí varios ejemplares; la contribución de los mismos autores, denominada 'Estudio Psychotechnico de algunas pruebas de aptitud', que ya ha sido debidamente apreciada por el Profesor Piéron en su 'Année Psychologique', la revisión de la serie de las pruebas de edad mental de Binet-Simon-Terman." (Lopes, 1932, p. 144, subrayado nuestro)

En uno de los editoriales de los Archivos de 1930, se destaca la importancia de la psicología y de sus métodos de evaluación por medio de pruebas psicológicas. Sobre esto, el editor afirma:

A este propósito nunca será bastante insistir sobre el enorme progreso que representa la moderna corriente de psicología de las pruebas mentales, usadas e interpretadas con el rigorismo necesario, para lo que mucho concurre el precioso auxilio de los procesos matemáticos, en su pluralidad transportados del campo de la estadística para el de las aplicaciones psicológicas. (Archivos Brasileños de Hygiene Mental, 1930, p. 312, subrayado nuestro)

Y sobre la relación entre la psicología y la higiene mental, añade:

(...) si existen métodos de realizar higiene mental, que en nuestro medio necesiten de estímulo y de incentivos para su perfeccionamiento, no son ellos por cierto, los métodos psiquiátricos, que los numerosos y proficientes especialistas nacionales podrán aplicar en cualquier momento, teniendo recursos materiales para hacerlo - sino los métodos psicológicos propiamente dichos, y de estos, sobre todo, los psicoexperimentales, cuantitativos y cualitativos, en la juiciosa clasificación de Claparède. Son estas técnicas que, más que cualquiera, permitirán al especialista la justa evaluación de las mentalidades normales, trabajo previo imprescindible a una serie de aplicaciones específicas de la higiene mental, en el sentido estricto, que decir concerniente a los individuos sanos de espíritu: orientación profesional, selección psicológica 
de los escolares, salud mental de los operarios, etc. (Archivos Brasileños de Hygiene Mental, 1930, p. 311 , subrayados nuestros)

En este caso, se entendía que la psicología era una ciencia a través de la cual sería posible conseguir la salubridad mental de la población, principalmente la población infantil, pretendiendo "el verdadero saneamiento psíquico" (Roxo, 1932, p. 89).

De esta forma, en las primeras décadas del siglo $\mathrm{XX}$, con la meta de mejorar el Brasil para equipararlo a las naciones desarrolladas, las acciones de los integrantes del movimiento higienista brasileño, como miembros de la LBHM, alineados al pensamiento científico de la época y amparados en los conocimientos psicológicos, tuvieron el objetivo de pensar e intervenir en la infancia como una de sus principales preocupaciones.

\section{La valoración del saber psicológico}

En relación con la defensa de la higiene mental como recurso, el Dr. Walclaw Radecki, como psicologista de la LBHM, la definía como "un conjunto de acciones prácticas, con el fin de crear condiciones que puedan facilitar el desarrollo psíquico de los individuos humanos, adaptando este desarrollo a las exigencias sociales y a la felicidad personal" (1925, p. 11).

En cuanto a la importancia atribuida a la psicología como medio para detectar la necesidad de establecer programas de higiene mental, él afirmaba:

La observación psicológica difiere, como sabemos, de la usada en otras ciencias naturales, por el hecho de que el acceso inmediato al fenómeno observado no es posible al investigador. Este puede observar las manifestaciones objetivas de las funciones psíquicas del investigado (palabras, acciones, cambios orgánicos), pero, el propio fenómeno psíquico se torna directamente "invisible". Solamente las analogías, que fijamos en las manifestaciones objetivas de las funciones, nos permiten concluir sobre su carácter. (Radecki, 1925, p. 13, subrayado nuestro seguido del resaltado del autor)
Con la psicología como soporte frente a la tarea de mejorar física y mentalmente a las futuras generaciones invirtiendo en la infancia, se atribuía a la higiene mental dos líneas de acción que fueron conceptuadas como:

(...) una, teniendo en cuenta el trabajo defensivo contra las causas de degeneración psíquica, es la profilaxis mental; otra, buscando preparar el equilibrio de adaptación entre la mentalidad individual y el medio físico y social, es la higiene mental propiamente dicha. (Fontenelle, 1925, p. 1, subrayado nuestro)

Otra ilustración con respecto a la importancia que los integrantes de la Liga atribuían a los conocimientos de psicología puede observarse, en la década de 1920, en relación con la formación de profesores; destacada por Helena Antipoff (18921974), psicóloga rusa radicada en Brasil en la década de 1920, cuando exponía en los Archivos las inversiones hechas por la Escuela de Perfeccionamiento de Profesores de Belo Horizonte - MG, en términos de capacitación en "psicología aplicada a la educación”. Tal inversión contaba, inclusive, con la contratación de profesores extranjeros para administrar una parte de las capacitaciones. Sobre tales contrataciones decía: "La asignatura de psicología, quedó entregue, en los tres primeros meses de la Escuela, al Dr. TH. Simon de Paris, y después, hasta el mes de agosto al profesor Leon Walther, de Genebra, pasando, finalmente, en septiembre de 1929, a la autora de estas líneas" (Antipoff, 1930, p. 227).

También registrando la forma como los higienistas brasileños se capacitaban para actuar como psicologistas y haciendo un análisis sobre las acciones de la LBHM en sus siete años de funcionamiento, en 1930 el Dr. Mirandolino Caldas escribió un editorial de los Archivos denominado "A Hygiene Mental no Brasil", por medio del cual hace un agarrado de las acciones desarrolladas y destaca la creación de un Laboratorio de Psicología Aplicada en el segundo semestre de 1926: "intensificándose cada vez más los trabajos de la Liga”. Al explicar cómo ocurrió tal implantación aclara que "para el fin de instalar el laboratorio y dar un curso de ini- 
ciación psicológica, fueron contratados, en Francia, por la Liga los psicologistas franceses profesor A. Fessard y Mme. A. B. Fessard, que aquí estuvieron por algunos meses" (Caldas, 1930, p. 73).

Otra forma de capacitación era la participación en congresos internacionales, de los cuales traían informaciones que buscaban socializar y poner en práctica. Ejemplo de esto fue la participación del Dr. Henrique Roxo en el IX Congreso Internacional de Psicología realizado en New-Haven-EE. UU., en 1929. Sobre tal evento, comenta los temas presentados y los evalúa como "asuntos interesantísimos". Y concluye: "sirviéndose principalmente de pruebas, los norte-americanos realizan un número tan grande de observaciones que mucha enseñanza proporcionarán a la humanidad" (Roxo, 1929, p. 138).

Este intercambio científico internacional también tenía lugar cuando recibían conferenciantes de otros países, tales como el Dr. J. Ramón Beltrán, que dictó algunas sobre psicología experimental en la República Argentina (Actas y Trabajos, 1930) o en forma de reseñas que se publicaban en una sección específica de Archivos. Se reseñaban diversos estudios e investigaciones nacionales e internacionales, de los cuales tomaban los conocimientos que juzgaban relevantes a la causa que defendían. Entre estas reseñas, en el área de la higiene mental infantil, podemos destacar títulos tales como: $\mathrm{La}$ organización de las clínicas psicológicas infantiles y de la vigilancia en el período evolutivo de Arnold Gesell (Lopes, 1930b), El desarrollo mental y la inteligencia de Henri Piéron (Leme Lopes, 1931), Es necesario comprender a los niños retrasados de Stanley Abbot (Caldas, 1931), Los orígenes del carácter en el niño de Henri Wallon (Caldas, 1934), entre otros.

La asociación entre la Liga y el saber psicológico se tornó más sólida cuando, en 1928, hubo la revisión de sus estatutos y el reconocimiento de que uno de sus objetivos debería ser el de "promover el estudio y el desarrollo de los nuevos procesos de la psicología aplicada y del psicoanálisis" (Estatutos de la Liga Brasileña de Higiene Mental, 1929, p. 40).

Por lo expuesto hasta el momento, así como Silva (2003), creemos que la psicología como uno de los instrumentos para la "promoción de higiene mental", proporcionó beneficios como también fue favorecida por el movimiento higienista, ya que encontró en Brasil un terreno fértil para su difusión como ciencia, aún en ascenso en el inicio del siglo XX.

\section{La Clínica de Euphrenia como un recurso}

Según los objetivos de los higienistas, conocer y proteger al niño como ser en formación podría garantizar el progreso y el futuro próspero que se anhelaba para la nación. En función de tal ideal, muchos de sus estudios demuestran la preocupación por aspectos de la vida del niño en lo que dice respecto, por ejemplo, al uso de pruebas psicológicas para evaluación de la inteligencia (Albuquerque, 1924), al suicidio de menores (Moncorvo Filho, 1930), al carácter del niño (Caldas, 1934), a los hurtos escolares (Ramos, 1934), al hijo único (Campos, 1934), entre otros.

Estas temáticas acrecentaron tanto la preocupación por la higiene mental del niño que en 1931 fue inaugurada la Clínica de Euphrenia "destinada a prevenir las enfermedades nerviosas de la infancia, corregir las reacciones psíquicas anormales y sublimar el carácter de la juventud". Creada por la LBHM, esta institución tenía el propósito de actuar como una clínica psicológica para niños, dentro del modelo de clínicas ya existentes en los Estados Unidos y Europa, como “(...) un servicio, no sólo con finalidades correctivas o de ajuste psíquico, sino también con objetivos constructivos, esto es, de perfeccionamiento del psiquismo, a través de una actuación médico-pedagógica directa en el período inicial del desarrollo mental infantil" (Archivos Brasileños de Hygiene Mental, 1932, p. 65).

Definiendo el objetivo principal de este servicio como el de "actuar en estos períodos importantes del desarrollo mental del niño", Caldas (1932, p. 32) explica la elección del término euphrenia (del griego, yo + phrenos, voluntad) como correlato a "fin de asegurar la buena formación del psiquismo". Con ocasión de la inauguración de la Clínica de Euphrenia, el médico Ernani Lopes (que era uno de sus directivos), en un discurso improvisado, destacó que los principios de la psicología elevarían la 
higiene mental al nivel de una ciencia, confiriéndole el papel de pedagogía del hábito o ciencia del comportamiento. En este caso la higiene mental

(...). Utilizándose de las conclusiones de las investigaciones realizadas en los más variados sectores, desde los experimentos de psicología comparada, sobre la adquisición y fijación de hábitos, hasta las advertencias preciosas del psicoanálisis sobre el proceso de evolución de la personalidad, ganó, poco a poco, realmente, la caracterología fueros de asignatura pragmática, de la cual tiene que valerse, muchas veces, el neuro-higienista, para el buen desempeño de sus tareas. De otras hechas es la caracterología la higiene mental misma, en una perfecta unificación de las técnicas respectivas. (Lopes, 1932, p. 78, subrayados nuestros)

Con esto, se esperaba que el niño atendido en la Clínica pudiera aprender

(...) no sólo a ser obediente, a comportarse bien, a tener buenas maneras, dotes clásicos de la educación tradicional, que se pagaba de apariencias, sino también a ser emprendedor, a tener espíritu de sacrificio, a ser alegre, solícito, cooperador, honesto, humanitario, una vez que se observe todo lo que preceptúa la higiene neuro-psíquica. (Lopes, 1932, p. 78)

En el acto solemne de la inauguración, el Dr. Ernani Lopes explicó a los presentes que, para fines de organizar los servicios que serían ofrecidos, se estableció un entendimiento entre la Liga y la Dirección General de Instrucción Pública de Rio de Janeiro. Sobre este hecho expresa:

(...) estamos listos para recibir a los escolares que nos quieran enviar, el Sr. Dr. Anísio Teixeira prontamente resolvió lo siguiente: que fueran enviados a la Liga no sólo los alumnos - problemas de todas las escuelas, sino todos los que tuvieran QP muy bajo o muy alto. Esta decisión muestra el alto criterio de aquella autoridad, pues en relación a los alumnos de QI muy alto evidentemente no se trata de casos anómalos debiendo, pues, ser prescriptas en esta especie medidas de pura euphrenia, en su sentido preciso, de higiene y no de terapéutica. (Lopes, 1932, p. 80, subrayado del autor)

Entre los servicios técnicos ofrecidos por la Clínica de Euphrenia, estaba el de psicología, donde la psicóloga jefe realizaba todo tipo de exámenes: pruebas de inteligencia, atención concentrada, atención difusa, atención asociada a la aptitud motora, raciocinio, sugestibilidad, afectividad, entre otros. Tales pruebas hacían parte de la evaluación que incluía examen físico y psíquico, además de investigación sobre el medio social del niño, con el fin de planificar la fase de "euphrenización" o de ajuste psíquico. En términos de resultados de esta evaluación, se percibe que la prueba psicológica tenía gran peso, ya que detectaba posibles desajustes que podrían comprometer el futuro del niño. Siendo así, en poco tiempo de funcionamiento de esta Clínica, ya se había constatado que la mayoría de niños presentaba

(...) desvios de la personalidad o inestabilidad afectiva, que necesitan ser corregidos. Son niños que, siendo amparados en el período crítico de su infancia, serán en el futuro los elementos y las dínamos, que accionarán el progreso de la patria, al paso que, desamparados o incomprendidos, podrán formar en la hilera de los psicópatas y criminales que constituyen el peso muerto de la nación. (Archivos Brasileños de Hygiene Mental, 1932, p. 77, subrayados nuestros)

El profesor Henrique Roxo, catedrático de psiquiatría y uno de los directivos de la Clínica, en entrevista al Periódico "O Globo" el 1 de noviembre de 1932, delimita la participación de varias áreas del saber, entre ellas la psicología, en la dinámica de la evaluación del niño, explicando que esta "se hace en tres fases sucesivas, en las cuales toman parte las enfermeras visitadoras, el psicologista y el neuro-higienista”. Y añade: "firmados los diagnósticos clínico, psicológico, y social, pasará el médico a la segunda parte de su tarea, esto es, a la terapéutica que será también clínica psicológica y social, pretendiendo, como había dicho, un verdadero, saneamiento psíquico" (Roxo, 1932, p. 89, subrayado nuestro). 
También el profesor Lourenço Filho, otro de sus directivos, concedió entrevista al periódico "O Globo" y así afirmó sobre el "convenio" entre la Liga y la educación:

El Dr. Anísio Teixeira, director de Instrucción, instituyó el servicio de "pruebas" y escalas, en todas las escuelas públicas del Distrito Federal, como una base necesaria a todos estos trabajos. La Liga de Hygiene Mental viene a su encuentro, para facilitar la parte del programa de psicología aplicada que excede del cuadro propiamente escolar. Son obras que se consagran para un fin altamente meritorio. (Lourenço Filho, 1932, p. 9 , resaltado del autor seguido de subrayado nuestro)

\section{La "euphrenia" en el espacio escolar}

A la medida que las ideas higienistas avanzaban en sus principios y formas de actuación, se instituían nuevos campos de inserción. Uno de ellos fue la inclusión de programas de higiene mental en las escuelas en asociación con las instituciones de instrucción. De este modo, "en la confluencia de las ideas sobre higiene mental y educación, surge la oportunidad de la instalación de clínicas de orientación infantil" (Mendonça, 2006, p. 55).

Al desarrollar un estudio bibliográfico sobre las clínicas de orientación infantil instaladas en escuelas públicas de Rio de Janeiro y de São Paulo, en la década de 30 del siglo XX, Mendonça (2006) describe la primera experiencia que ocurrió en Rio de Janeiro, donde tal clínica fue organizada por el médico higienista Arthur Ramos en 1931, a partir de servicios ya existentes en las escuelas primarias que servían "de campo de investigación y experimentación para el Servicio de Psicología Aplicada del Instituto de Educación y para los estudios e investigaciones del Servicio de Higiene y Educación Sanitaria Escolar" (p. 55). En São Paulo, como una segunda experiencia, esta iniciativa se dio en 1938 a manos del médico higienista, Durval Marcondes, sobre la cual explica que:

Al examen médico general y especializado, así como a la investigación psicológica de laboratorio, se combina, pues, en la clínica, el estudio de la historia personal y social del niño, estudio este a encargo de la visitadora psiquiátrica, una de las piezas fundamentales de la organización y como que su prolongamiento en el seno de la comunidad. (Marcondes, 1941a, p. 5 mencionado por Mendonça, 2006, p. 61)

\section{Consideraciones finales}

Iniciamos esta reflexión, afirmando que la creación de servicios para atender la infancia en la contemporaneidad puede tomarse como una novedad y nos propusimos demostrar que, en realidad, tal proyecto ya hacía parte de las acciones de los integrantes de la LBHM, en las primeras décadas del siglo XX. Aún así, nos propusimos señalar evidencias de lo que en muchos análisis es ignorado: la inserción de la psicología en Brasil a manos de este grupo que aspiraba al progreso, identificado en naciones consideradas por ellos como desarrolladas y, por lo tanto, modelos que debían seguirse.

De este hecho, a partir de las primeras décadas del siglo XX, florecieron en Brasil, ideas e ideales que consideraban la higiene mental un medio de mejorar psíquicamente a la población. En este proceso, la psicología fue llamada a intervenir lo más precozmente posible en la infancia, con la pretensión de "preparar el equilibrio de adaptación entre la mentalidad individual y el medio psíquico y social". Se iniciaba allí un proceso continuo de difusión del saber psicológico amparado en bases científicas, construido, hasta ese momento, sobre nuevos saberes con la visión de hombre y de mundo de estos hombres de ciencia que tenían en la prevención de las enfermedades mentales, vía higiene mental, una forma de evitar "fracasos de ajuste" que podrían "desnaturalizar la vida y profundamente complicar la eficiencia y felicidad del individuo" (Fontenelle, 1925, p. 1-2). Para ello, tenían en la familia y en la escuela algunos de sus principales enfoques de intervención.

Hoy, pasado casi un siglo, tenemos noticia de experiencias que nos remiten a las acciones tan defendidas por los higienistas que, por una visión menos atenta, se atribuyen a un pasado distante y ya superado. El enfoque en la prevención o detección precoz, estipulado en un programa de la Universi- 
dad Federal de São Paulo (UNIFESP) en 2009, sirve para recordar tales acciones, ya que prevé:

(...) que médicos y otros profesionales de la salud vayan hasta las escuelas a enseñar a los profesores a identificar alumnos con sospecha de enfermedades psiquiátricas graves, como la esquizofrenia. El enfoque son estudiantes entre 11 y 18 años de 40 escuelas públicas de São Paulo [...] el objetivo es la detección precoz. Los profesores pueden ayudar mucho en la identificación de señales sugestivas [de la enfermedad]. A veces, los adolescentes pasan más tiempo con ellos que con sus padres. (Collucci, 2009, subrayados nuestros)

Un poco menos distante del cotidiano de los profesionales de la salud y de la educación fue la iniciativa de la $15 .{ }^{a}$ Regional de Salud de Maringá, en el Estado de Paraná (2014) que recientemente ha promovido un evento de capacitación denominado "Oficina de Sensibilización Voces del SUS - Identificación precoz de riesgo y sufrimiento psíquico en bebés", con el objetivo de "sensibilizar a profesionales de la salud y de la educación" para la "detección precoz de señales de riesgo y de sufrimiento psíquico en bebés, bajo la perspectiva de las intervenciones de prevención en el área de Salud Mental que colaboren para la no instalación de cuadros psicopatológicos" o aun para que disturbios ya diagnosticados no se agraven.

Ejemplos como este pueden configurar una de las iniciativas actuales de detectar precozmente perjuicios que podrían manifestarse en etapas posteriores del desarrollo de los individuos. Con la distancia que el tiempo transcurrido permite, es posible afirmar que este era uno de los principales objetivos de los higienistas. En otras palabras, ellos buscaban realizar el "saneamiento psíquico" o la "psicohigiene", utilizando las herramientas de que disponían: el saber psicológico. Para ello idealizaron, entre otros servicios, la clínica de "euphrenia" y las clínicas de orientación infantil.

Además de iniciativas específicas como estas, actualmente, también contamos con varias entidades que tienen como objetivo acoger demandas de salud mental, como el CAPSi (Centro de
Atención Psicosocial Infantojuvenil), mencionado anteriormente. Este Centro, de acuerdo con el Decreto N.. 336 (2002) del Ministerio de la Salud, tiene como objetivo promover la atención a niños y adolescentes con trastornos mentales, por medio de acciones de carácter individual (medicamentos, psicoterapéutico, de orientación, entre otros); en grupos (psicoterapia, grupo operativo, actividades de soporte social, entre otros); oficinas terapéuticas; visitas y atenciones domiciliarias; atención a la familia; actividades comunitarias enfocando la integración del niño y del adolescente en la familia, en la escuela, en la comunidad o cualesquiera otras formas de inserción social; acciones intersectoriales, principalmente con las áreas de asistencia social, educación y justicia; entre otras. El equipo técnico debe estar compuesto por un médico (psiquiatra o neurólogo) y un enfermero, y con más de cuatro profesionales de las siguientes categorías: psicólogo, asistente social, enfermero, terapeuta ocupacional, fonoaudiólogo, pedagogo u otro profesional necesario al proyecto terapéutico.

Salvaguardando las debidas particularidades o peculiaridades que van más allá de cada tiempo histórico, podemos identificar señales de proposiciones de los higienistas en programas y propuestas actuales que, al basarse en la concepción de prevención o identificación precoz de señales de riesgo, pretenden promover la psicohigiene infantil. Sin embargo, en gran medida, las herramientas de la actualidad son otras y, quizás, muy polémicas: los psicotrópicos. Pero, esto es otra historia...

\section{Referencias}

Actas e trabalhos da Liga Brasileira de Higiene Mental. (1930). Conferência do professor J. Ramon Beltran sobre a psicologia na Argentina. Trabalhos originais. Arquivos Brasileiros de Higiene Mental, 3(8), 305-310.

Albuquerque, M. (1925). Testes: introdução ao estudo dos meios científicos de julgar a inteligência e a aplicação dos alunos. Resenhas e Analyses. Arquivos Brasileiros de Higiene Mental, 1(1), 183-184.

Antipoff, H. (1930). A psicologia na escola de aperfeiçoamento de Belo Horizonte. Trabalhos origi- 
nais. Arquivos Brasileiros de Higiene Mental, 3(7), 226-234.

Arquivos Brasileiros de Higiene Mental. (1930). [Editorial]. 4(1-2-3), 311-312.

Arquivos Brasileiros de Higiene Mental. (1932). A Clínica de Euphrenia, 5(2), 65-98.

Boarini, M. L. (2012). Apresentação. En M. L. Boarini (Org.), Higiene mental: ideias que atravessaram o século XX (pp. 11-24). Maringá: Eduem.

Brasil, Informativo eletrônico de dados sobre a Política Nacional de Saúde Mental. (2012). Recuperado de http://saudeecosol.files.wordpress.com/2012/03/ saude-mental-em-dados-10-ms.pdf

Brasil, Ley 4.119 de 1962. Dispõe sobre os cursos de formação em Psicologia e regulamenta a profissão de Psicólogo. Agosto 27 de 1962. DO 9253. Recuperado de http://www.pol.org.br/legislacao/pdf/ lei_n_4.119.pdf

Brasil, Ministério da Saúde. (2012). Coordenação Geral de Saúde Mental, Álcool e Outras Drogas [Saúde Mental em Dados - 10, Ano VII, n. 10, março de 2012].

Brasil, Porteria N.. 336 de 2002 [Ministério da Saúde]. Estabelece que os Centros de Atenção Psicossocial poderão constituir-se nas modalidades de serviços: CAPS I, CAPS II e CAPS III, definidos por ordem crescente de porte/complexidade e abrangência populacional. Febrero 19 de 2002. Recuperado de http://portal.saude.gov.br/portal/arquivos/pdf/ Portaria\%20GM\%20336-2002.pdf

Brasil, Secretaria da Saúde. (2014). 15ª Regional de Saúde de Maringá, Paraná. Oficina de Sensibilização Vozes do SUS - Identificação precoce de risco e sofrimento psíquico em bebês. Recuperado de https://docs.google.com/forms/d/ 1wK_PntCEjONnDRCnxXI95GR4yfg8ReW7h0NKeNDCZ5s/ viewform?sid\&c $=0 \& w=1 \&$ token

Caldas, M. (1929). Os Arquivos Brasileiros de Higiene Mental. Arquivos Brasileiros de Higiene Mental, 2(1), 1-2.

Caldas, M. (1930). A higiene mental no Brasil. Arquivos Brasileiros de Higiene Mental, 3(3), 69-77.

Caldas, M. (1931). Stanley Abbot. E' preciso comprehender as crianças atrasadas. Resenhas e analyses. Arquivos Brasileiros de Higiene Mental, 4(1), 95-96.
Caldas, M. (1932). A euphrenia: ciência da boa celebração. Arquivos Brasileiros de Higiene Mental, 2(1), 29-40.

Caldas, M. (1934). Henri Wallon. As origens do caráter na criança. Resenhas e analyses. Arquivos Brasileiros de Higiene Mental, 7(1), 36-37.

Campos de, M. (1934). O filho único e a higiene mental. Trabalhos originais. Arquivos Brasileiros de Higiene Mental, 2(1), 3-17.

Campos, R. H. de F. (Org.). (2001). Dicionário biográfico da psicologia no Brasil (1.a ed.). Rio de Janeiro: Imago.

Centofanti, R. (1982). Radecki e a psicologia no Brasil. Psicologia: Ciência e Profissão, 3(1), 2-50.

Collucci, C. (2 de junio de 2009). Professor é educado para identificar esquizofrenia. Folha de S. Paulo. Recuperado de http://www1.folha.uol.com.br/fsp/ saude/sd0206200901.htm

Costa, J. F. (1989). História da psiquiatria no Brasil: um corte ideológico (4.a ed.). Rio de Janeiro: Xenon.

Costa, J. F. (2004). Ordem médica e norma familiar (5. ed.). Rio de Janeiro: Edições Graal.

Estatutos da Liga Brasileira de Higiene Mental. (1929). Arquivos Brasileiros de Higiene Mental, 2(1), 39-47.

Feitosa, J. B. (2012). A institucionalização do adolescente autor de infração. En M. L. Boarini (Org.), Higiene mental: ideias que atravessaram o século XX (pp.79-109). Maringá: Eduem.

Fontenelle, J. P. (1925). Higiene mental e educação. Arquivos Brasileiros de Higiene Mental, 1(1), 1-10.

Leme Lopes, M. B. (1931). Henri Piéron. O desenvolvimento mental e a inteligência. Resenhas e analyses. Arquivos Brasileiros de Higiene Mental, 4(1), 65-68.

Lourenço Filho, M. B. [Entrevista al periódico O Globo el 7 de noviembre de1932]. Arquivos Brasileiros de Higiene Mental, 5(2), 89-91.

Lopes, E. (1930a). Menores incorrigíveis. Arquivos Brasileiros de Higiene Mental, 3(7), 241-246.

Lopes, E. (1930b). A organização das clínicas psicológicas infantis e da vigilância no período evolutivo de Arnold Gesell. Arquivos Brasileiros de Higiene Mental, 3(4), 241-242.

Lopes, E. (1932). A clínica de euphrenia. Arquivos Brasileiros de Higiene Mental, 5(2), 65-98. 
Massimi, M. (1990). História da psicologia brasileira. Da época colonial até 1934. São Paulo: Editora Pedagógica e Universitária Ltda.

Massimi, M. (2001). A psicologia dos Jesuítas: uma contribuição à história das ideias psicológicas. Psicologia: Reflexão e Crítica, 14(3), 625-633.

Mendonça, D. (2006). A higiene mental do escolar: o ardil da ordem (Dissertação de mestrado nãopublicada). Universidade Estadual de Maringá, Maringá, Brasil.

Moncorvo Filho, A. (1930). Suicídio de menores. Arquivos Brasileiros de Higiene Mental, 3(7), 167-176.

Radecki, W. (1925). Higiene mental da criança: baseada sobre as leis da psicologia. Arquivos Brasileiros de Higiene Mental, 1(1), 11-89.

Ramos, A. (1934). Os furtos escolares. Trabalhos Originais. Arquivos Brasileiros de Higiene Mental, 7(3), 229-235.

Reis, J. R. F. (2003). Degenerando em barbárie: a hora e a vez do eugenismo radical. En M. L. Boarini (Org.),
Higiene e raça como projetos: higienismo e eugenismo no Brasil (pp.133-164). Maringá: Eduem.

Roxo, H. (1929). Impressões do congresso de psicologia de New-Haven. Arquivos Brasileiros de Higiene Mental, 2(1), 134-138.

Roxo, H. (1932). [Entrevista al periódico O Globo el 1.o de noviembre de 1932]. Arquivos Brasileiros de Higiene Mental, 5(2), 88-89.

Silva da, L. C. (2003). A contribuição da higiene mental para o desenvolvimento da psicologia no Brasil. En M. L. Boarini (Org.), Higiene e raça como projetos: higienismo e eugenismo no Brasil (pp.133-164). Maringá: Eduem.

Zaniani, E. J. M., \& Boarini, M. L. (2011). Infância e vulnerabilidade: repensando a proteção social. Psicologia Eु Sociedade, 23(2), 272-281. Recuperado de http://www.scielo.br/scielo.php?pid=S01027182 $2011000200008 \&$ script $=$ sci arttext 